\title{
Cosmic Far-Infrared Background Radiation
}

\section{Vladimir S. Netchitailo}

Biolase Inc.

4 Cromwell, Irvine CA 92618, USA

E-mail:v.netchitailo@sbcglobal.net

In this manuscript, we discuss mass-varying neutrinos and propose their energy density to exceed that of baryonic and dark matter. We introduce cosmic Large Grains whose mass is about Planck mass, and their temperature is around $29 \mathrm{~K}$. Large grains are in fact Bose-Einstein condensates of proposed dineutrinos, and are responsible for the cosmic Far-Infrared Background radiation.

XII Multifrequency Behaviour of High Energy Cosmic Sources Workshop 12-17 June, 2017

Palermo, Italy 


\section{Introduction}

Hypersphere World - Universe Model (WUM) views the World as a 3-dimensional Hypersphere that expands along the fourth spatial dimension in the Universe. The hypersphere as a model of finite universe was proposed by Georg Riemann in 1854 [1], [2].

WUM is based on Maxwell's equations that form the foundation of Electromagnetism and Gravitoelectromagnetism that was proposed by O. Heaviside in 1893. Gravitoelectromagnetism is an approximation to the Einstein's field equations for General Relativity in the weak field limit. According to Maxwell's equations, there are two measurable physical characteristics: energy density and energy flux density [3].

WUM introduces a fundamental dimensionless time-varying parameter $Q$ that is the measure of the curvature of the Hypersphere $R$. It can be calculated from the value of the gravitational constant $G$ and in present epoch equals to [4]:

$$
Q=\frac{R}{a}=v_{0} \tau=\frac{a^{2} c^{4}}{8 \pi h c} \times G^{-1}=0.759972 \times 10^{40}
$$

where $a=2 \pi a_{0}$ is a basic unit of length and $a_{0}$ is the classical electron radius; $v_{0}=c / a$ is a basic unit of frequency; $c$ is the gravitoelectrodynamic constant that is identical to the electrodynamic constant $c$ in Maxwell's equations; $\tau$ is a cosmological time; and $h$ is Planck constant.

\section{Cosmic Neutrinos Background}

The existence of the World's Medium is a principal point of WUM. It follows from the observations of Intergalactic Plasma and Cosmic Microwave Background Radiation [5]; Far-Infrared Background Radiation and Cosmic Neutrino Background [6]; Gamma-ray Background Radiation [7]. The Medium consists of stable elementary particles with lifetimes longer than the age of the World: protons with mass $m_{p}$, electrons with mass $m_{e}$, neutrinos with mass $m_{v}$, photons, and dark matter particles [7].

Cosmic Neutrino Background (CNB) consists of three different types of neutrinos: electronic $v_{e}$, muonic $v_{\mu}$, and tauonic $v_{\tau}$, and their antiparticles. Pontecorvo and Smorodinskii discussed the possibility of energy density of neutrinos exceeding that of baryonic matter [8]. Neutrino oscillations imply that neutrinos have non-zero masses.

In WUM, neutrino masses are related to and proportional to basic unit of mass $m_{0}=h / a c$ multiplied by fundamental parameter $Q^{-1 / 4}$ and different coefficients. Neutrinos exist in superposition of the following mass eigenstates predicted by WUM [6]:

$$
\begin{aligned}
& m_{v_{e}}=\frac{1}{24} m_{0} \times Q^{-1 / 4}=3.1250 \times 10^{-4} \mathrm{eV} / \mathrm{c}^{2} \\
& m_{v_{\mu}}=m_{0} \times Q^{-1 / 4}=7.4999 \times 10^{-3} \mathrm{eV} / \mathrm{c}^{2} \\
& m_{v_{\tau}}=6 m_{0} \times Q^{-1 / 4}=4.5000 \times 10^{-2} \mathrm{eV} / \mathrm{c}^{2}
\end{aligned}
$$


The squared values of the muonic and tauonic neutrinos masses fall into the ranges of mass splitting $\Delta m_{\text {sol }}^{2}$ and $\Delta m_{a t m}^{2}$ for solar and atmospheric neutrinos respectively. One of the principal ideas of WUM holds that energy densities of Medium particles are proportional to proton energy density in the World's Medium:

$$
\Omega_{p}=\frac{2 \pi^{2} \alpha}{3}=0.048014655
$$

which depends on the constant $\alpha=m_{e} / m_{0}$. Therefore, the total neutrinos relative energy density $\Omega_{v t o t}$ of the CNB in terms of the critical energy density $\rho_{c r}$ equals to [6]:

$$
\Omega_{v t o t}=\frac{45}{\pi} \Omega_{p}=30 \pi \alpha=0.68775927
$$

The total neutrinos energy density in the World $\Omega_{v t o t}$ is almost 15 times greater than the baryonic energy density in the Medium: $\Omega_{v t o t} \cong 15 \Omega_{p}$. At such a high neutrinos concentration, "neutrinos pairs" $v \bar{v}$ (dineutrinos) can be created. Bellow we will discuss their role in Far-Infrared Background radiation.

One may wonder - if there are so many neutrinos out there, how come the numerous neutrino detectors do not register them in significant quantities? The answer on this question follows from the calculations of neutrinos energies: the CNB consists of very low-energy neutrinos, whose energy is similar to that of the Cosmic Microwave Background radiation. Their interaction with matter is very weak. Since the neutrino-induced cross-sections depend on the neutrinos energy, such background neutrinos will not be registered by standard neutrino detectors. In fact, we might never be able to directly observe the CNB.

\section{Cosmic Far-Infrared Background}

The cosmic Far-Infrared Background (FIRB), which was announced in January 1998, is part of the Cosmic Infrared Background, with wavelengths near 100 microns that is the peak power wavelength of the black body radiation at temperature $29 \mathrm{~K}$ [9]. We introduce Bose-Einstein Condensate (BEC) drops of dineutrinos whose mass is about Planck mass, and their temperature is around $29 \mathrm{~K}$. These drops are responsible for the FIRB [6].

According to the literature [10], [11], [12], the size of large cosmic grains $D_{G}$ is roughly equal to the length $L_{F}$ in WUM:

$$
D_{G} \sim L_{F}=a \times Q^{1 / 4}=1.6532 \times 10^{-4} \mathrm{~m}
$$

and their mass $m_{G}$ is close to the Planck mass $M_{P}=2.17647 \times 10^{-8} \mathrm{~kg}$ :

$$
m_{G} \sim\left(10^{-9} \Leftrightarrow 10^{-7}\right) \mathrm{kg}
$$


The calculated density of grains $\rho_{G}$ is about:

$$
\rho_{G} \sim \frac{6}{\pi} \frac{M_{P}}{L_{F}^{3}} \approx 9.2 \times 10^{3} \mathrm{~kg} / \mathrm{m}^{3}
$$

According to WUM, Planck mass $M_{P}$ equals to

$$
M_{P}=2 m_{0} \times Q^{1 / 2} \propto \tau^{1 / 2}
$$

Note that the value of $M_{P}$ is increasing with cosmological time, and is proportional to $\tau^{1 / 2}$.

A grain of mass $B_{1} M_{P}$ and radius $B_{2} L_{F}$ is receiving energy from the Medium of the World as the result of dineutrinos Bose-Einstein condensation at the following rate:

$$
\frac{d}{d \tau}\left(B_{1} M_{P} c^{2}\right)=\frac{B_{1} M_{P} c^{2}}{2 \tau}
$$

where $B_{1}$ and $B_{2}$ are parameters. The received energy will increase the grain's temperature $T_{G}$, until equilibrium is achieved: power received equals to the power irradiated by the surface of a grain in accordance with the Stefan-Boltzmann law:

$$
\frac{B_{1} M_{P} c^{2}}{2 \tau}=\sigma_{S B} T_{G}^{4} \times 4 \pi B_{2}^{2} L_{F}^{2}
$$

where $\sigma_{S B}$ is Stefan-Boltzmann constant:

$$
\sigma_{S B}=\frac{2 \pi^{5} k_{B}^{4}}{15 h^{3} c^{2}}
$$

With Nikola Tesla's principle at heart - There is no energy in matter other than that received from the environment - we get:

$$
B_{1} M_{P} c^{2}=4 \pi B_{2}^{2} L_{F}^{2} \sigma_{0}
$$

where $\sigma_{0}=\frac{h c}{a^{3}}$ is a basic unit of surface energy density. We then calculate the grain's stationary temperature $T_{G}$ to be

$$
T_{G}=\left(\frac{15}{4 \pi^{5}}\right)^{1 / 4} \frac{h c}{k_{B} L_{F}}=28.955 \mathrm{~K}
$$

This result is in an excellent agreement with experimentally measured value of $29 \mathrm{~K}$. Cosmic FIRB radiation is not a black body radiation. Otherwise, its energy density $\rho_{F I R B}$ at temperature $T_{G}$ would be too high and equal to the energy density of the Medium of the World. The total flux of the FIRB radiation is the sum of the contributions of all individual grains [6]. 


\section{Bose-Einstein Condensate}

New cosmological models employing the Bose-Einstein Condensates (BEC) have been actively discussed in literature in recent years [6]. The transition to BEC occurs below a critical temperature $T_{c}$, which for a uniform three-dimensional gas consisting of non-interacting particles is given by [13]:

$$
T_{C}=[\zeta(3 / 2)]^{-2 / 3} \frac{h^{2} n_{X}^{2 / 3}}{2 \pi m_{X} k_{B}} \approx \frac{h^{2} n_{X}^{2 / 3}}{11.918 m_{X} k_{B}}
$$

where $n_{X}$ is the bosons' concentration, $m_{X}$ is the mass per boson, $\zeta$ is the Riemann zeta function: $\zeta(3 / 2) \approx 2.6124$. According to our Model, we can take the value of the critical temperature $T_{c}$ to equal the stationary temperature $T_{G}$ of large grains. Let's assume that the energy density of boson particles $\rho_{X}$ equals to the Microwave Background Radiation (MBR) energy density $\rho_{M B R}[6]$ :

$$
\rho_{X}=n_{X} m_{X}=\rho_{M B R}=4 \pi^{2} \alpha \frac{m_{e}}{m_{p}} \frac{h c}{L_{F}^{4}}=1.5690 \times 10^{-4} \times \frac{h c}{L_{F}^{4}}
$$

Considering equations (3.9), (4.1) and (4.2), we can calculate the value of $n_{X}$ :

$$
\begin{aligned}
& n_{X}=\left[47.672 \pi^{2} \alpha \frac{m_{e}}{m_{p}}\left(\frac{15}{4 \pi^{5}}\right)^{1 / 4}\right]^{3 / 5} \times L_{F}^{-3}= \\
& =0.011922 \times L_{F}^{-3}=2.6386 \times 10^{9} \mathrm{~m}^{-3}
\end{aligned}
$$

and the value of the mass $m_{X}$ :

$$
m_{X}=\frac{\rho_{X}}{n_{X} c^{2}}=0.013161 \times m_{0} \times Q^{-1 / 4}=0.987 \times 10^{-4} \mathrm{eV} / \mathrm{c}^{2}
$$

The mass of the dineutrinos $m_{X}$ is in the range of electronic neutrinos mass (see (2.1)).

The calculated values of mass and concentration of dineutrinos satisfy the conditions for their Bose-Einstein condensation. Consequently, BEC drops whose masses are about Planck mass can be created. Dineutrinos stability follows from the calculation of the maximum kinetic energy of tauonic neutrinos in the Cosmic Neutrinos Background which is $144 \pi^{2}$ less than the rest energy of tauonic neutrinos [1]. The stability of BEC drops is provided by the detailed equilibrium between the energy absorption from the Medium of the World (provided by dineutrinos because of their Bose-Einstein condensation) and re-emission of this energy in FIRB at the stationary temperature $T_{G} \approx 29 \mathrm{~K}$.

To summarize,

Bose-Einstein Condensate drops of dineutrinos are responsible for FIRB:

- Drop masses about equal to Planck mass;

- $\quad$ Temperature around 29K.

WUM explanation of FIRB is in good agreement with experimentally measured characteristics. 


\section{Acknowledgements}

Special thanks to Prof. Franco Giovannelli for extending an invitation to the Workshop.

\section{References}

[1] V. S. Netchitailo, Overview of Hypersphere World-Universe Model, Journal of High Energy Physics, Gravitation and Cosmology 2 (2016) 593.

[2] V. S. Netchitailo, Mathematical Overview of Hypersphere World-Universe Model, Journal of High Energy Physics, Gravitation and Cosmology 3 (2017) 15.

[3] V. S. Netchitailo, 5D World-Universe Model. Space-Time-Energy, Journal of High Energy Physics, Gravitation and Cosmology 1 (2015) 25.

[4] V. S. Netchitailo, 5D World-Universe Model. Gravitation, Journal of High Energy Physics, Gravitation and Cosmology 2 (2016) 328.

[5] V. S. Netchitailo, Burst Astrophysics, Journal of High Energy Physics, Gravitation and Cosmology 3 (2017) 157.

[6] V. S. Netchitailo, 5D World-Universe Model. Neutrinos. The World, Journal of High Energy Physics, Gravitation and Cosmology 2 (2016) 1.

[7] V. S. Netchitailo, 5D World-Universe Model. Multicomponent Dark Matter, Journal of High Energy Physics, Gravitation and Cosmology 1 (2015) 55.

[8] B. Pontecorvo and Y. Smorodinsky, The Neutrino and the Density of Matter in the Universe, Sov. Phys. JETP 14 (1962) 173.

[9] G. Lagache, et al., First detection of the Warm Ionized Medium Dust Emission. Implication for the Cosmic Far-Infrared Background, arXiv:9901059.

[10] M. Maurette, J. Cragin, and S.Taylor, Cosmic Dust in 50 KG Blocks of Blue Ice from Cap-Prudhomme and Queen Alexandra Range, Antarctica, Meteoritics 27 (1992) 257.

[11] J. M. Saxton, S. F. Knotts, G. Turner, and M. Maurette, 40Ar/39Ar Studies of Antarctic Micrometeorites, Meteoritics 27 (1992) 285.

[12] A. A. Jackson and H. A. Zook, Dust Particles from Comets and Asteroids: Parent-Daughter Relationships, Abstracts of the Lunar and Planetary Science Conference 22 (1991) 629.

[13] L. D. Landau and E. M. Lifshitz, Statistical Physics, Third Edition 5 (1980). 